\title{
Analysis of Questionnaire Investigation on SNS Utilizing Bayesian Network
}

\author{
Tsuyoshi Aburai * \\ Graduate School of Policy and Management, Doshisha University, Kyoto, Japan \\ Yuki Higuchi \\ Faculty of Business Administration, Setsunan University, Osaka, Japan \\ Kazuhiro Takeyasu \\ College of Business Administration, Fuji Tokoha University, Shizuoka, Japan
}

(Received: October 28, 2012 / Revised: March 1, 2013 / Accepted: May 16, 2013)

\begin{abstract}
Social Networking Service (SNS) is prevailing rapidly in Japan in recent years. The most popular ones are Facebook, mixi, and Twitter, which are utilized in various fields of life together with the convenient tool such as smart-phone. In this work, a questionnaire investigation is carried out in order to clarify the current usage condition, issues and desired functions. More than 1,000 samples are gathered. Bayesian network is utilized for this analysis. After conducting the sensitivity analysis, useful results are obtained. Differences in usage objectives and SNS sitesare made clear by the attributes and preference of SNS users. They can be utilized effectively for marketing by clarifying the target customer through the sensitivity analysis.
\end{abstract}

Keywords: SNS, Questionnaire Investigation, Bayesian Network

* Corresponding Author, E-mail: aburai79@gmail.com

\section{INTRODUCTION}

Social Networking Service (SNS) is prevailing rapidly in recent years. Facebook, mixi, and Twitter are the most popular ones. It is well known that Facebook played an important role in communication under the condition that the telephones and/or cellular phones connected with Internet could not make links when the big disaster hit the eastern part of Japan. Google launched forth into SNS by the name Google+ in June 2011. Thus, it has become a hot business spot and it is exerting great influence upon society and economy. In this paper, a questionnaire investigation is conducted in order to clarify the current usage condition, issues and desired functions.

Differences in usage objectives and SNS sites would be made clear by the attributes and preference of SNS users.
For these purposes, we created a questionnaire investigation of jewelry/accessory purchasing (SNS). In recent years, the Bayesian network is highlighted because it has the following good characteristics (Neapoli$\tan , 2004)$.

- Structural Equation Modeling requires normal distribution to the data in the analysis. Therefore, it has a limitation in making analysis, but the Bayesian network does not require a specific distribution type to the data. It can handle any distribution type.

- It can handle the data which include partial data.

- Expert's know-how can be reflected in building a Bayesian Network model.

- Sensitivity analysis can be easily performed by settling evidence. We can estimate and predict the prospective purchaser by that analysis. 
- It is a probability model having a network structure. Related items are connected with directional link. Therefore, understanding becomes easy by its visual chart.

This research utilizes the Bayesian network to analyze SNS users' current usage conditions, issues and desired functions because no variable is required to have normal distribution. Reviewing past researches, there are some related researches as follows. Tsuji et al. (2008) have analyzed preference mining on future home energy consumption. There are some papers concerning purchase behavior in the shop (Tatsuoka et al., 2008a, 2008b), but no research has been reported on the SNS users utilizing Bayesian network.

Bayesian network is utilized for this analysis. After executing the sensitivity analysis, useful results are obtained. Differences in usage objectives and SNS sites are made clear by the attributes and preference of SNS users. It can be utilized effectively for marketing by clarifying the target customer through the sensitivity analysis.

The rest of the paper is organized as follows. The outline of questionnaire research is stated in Section 2. In Section 3, an analysis by hypothesis testing is executed. In section 4, Bayesian network analysis is executed which is followed by the sensitivity analysis in Section 5. Section 6 is a summary.

\section{OUTLINE OF QUESTIONNAIRE RESEARCH AND EXAMINEES}

\subsection{Outline of Questionnaire Research}

We make a questionnaire investigation concerning the SNS. Outline of questionnaire research is as follows. (1) Scope of investigation : student, government employee, and company employee, etc., Japan

(2) Period

(3) Method

(4) Collection

: April/26/2012-June/6/2012

: mail, online and self-writing

: number of distribution

1,500 ; number of collection

1,197 (collection rate $79.8 \%$ );

Valid answer 1,098

Analysis methods are as follows.

Questionnaire results are analyzed in three ways. First, analysis by hypothesis testing is executed inSection3 in order to confirm the hypotheses. Second, analysis by Bayesian Network is executed in Section4 in order to clarify and visualize the causal relationship among the items. Third, analysis by sensitivity analysis is executed in Section 5 in order to predict the prospective purchaser as is shown in Table1.
Table 1. Analysis procedure

\begin{tabular}{cll}
\hline Step & \multicolumn{1}{c}{ Aim of analysis } & \multicolumn{1}{c}{ Used method } \\
\hline (1) & Confirm hypotheses & Hypothesis testing \\
(2) & $\begin{array}{l}\text { Build Bayesian network in or- } \\
\text { der to clarify and visualize the }\end{array}$ & $\begin{array}{l}\text { Bayesian network } \\
\text { analysis }\end{array}$ \\
& $\begin{array}{l}\text { causal relationship among } \\
\text { items }\end{array}$ & \\
(3) & $\begin{array}{l}\text { Predict the prospective pur- } \\
\text { chaser }\end{array}$ & Sensitivity analysis \\
\hline
\end{tabular}

\subsection{Outline of Examinees}

Table 2. Major single variable summary results

\begin{tabular}{|c|c|}
\hline Questionnaire & No. of answer $(\%)$ \\
\hline \multicolumn{2}{|l|}{ Q1. Use the SNS } \\
\hline Use & $792(72.1)$ \\
\hline Do not use & $306(27.9)$ \\
\hline \multicolumn{2}{|l|}{ Q13. Gender } \\
\hline Male & $650(59.2)$ \\
\hline Female & $448(40.8)$ \\
\hline \multicolumn{2}{|l|}{ Q14.Age } \\
\hline$<20$ & $196(17.9)$ \\
\hline $21 \sim 30$ & $328(29.9)$ \\
\hline $31 \sim 40$ & $299(27.2)$ \\
\hline $41 \sim 50$ & $194(17.7)$ \\
\hline $51 \sim 60$ & $73(6.6)$ \\
\hline$>60$ & $8(0.7)$ \\
\hline \multicolumn{2}{|l|}{ Q15. Occupation } \\
\hline Student & 295 (26.9) \\
\hline Government employee & $15(1.4)$ \\
\hline Company employee & $595(54.2)$ \\
\hline Schoolteacher/staff & $43(3.9)$ \\
\hline Clerk of organization & $19(1.7)$ \\
\hline Independents & $45(4.1)$ \\
\hline Temporary employee & $15(1.4)$ \\
\hline Part-timers & $53(4.8)$ \\
\hline Miscellaneous & $18(1.6)$ \\
\hline \multicolumn{2}{|l|}{ Q16. Residence } \\
\hline Hokkaido & $22(2.0)$ \\
\hline Tohoku region & $49(4.5)$ \\
\hline Kanto region & $157(14.3)$ \\
\hline Chubu region & $176(16.0)$ \\
\hline Kansai region & $400(36.4)$ \\
\hline Chugoku region & $110(10.0)$ \\
\hline Shikoku region & $105(9.6)$ \\
\hline Kyushu region & $79(7.2)$ \\
\hline
\end{tabular}

\section{THE RESULTS OF STATISTICAL HYPOTHESIS TESTING}

\subsection{Outline of Questionnaire Research}

$\chi^{2}$ hypothesis testing is executed on users' SNS 
consciousness. $\chi^{2}$ hypothesis testing is to clarify the difference between the expected value and the observed data, which is shown in Eq. (1)

$$
\chi^{2}=\sum_{i=1}^{n} \frac{\left(O_{i}-E_{i}\right)^{2}}{E_{i}}
$$

where $O_{i}$ is an observed data and $E_{i}$ is an expected value.

Hypotheses are built based upon the following viewpoints (Table 3 ).

Table 3. Hypothesis building viewpoints (source: Kotleret al., 2012; revised by the writer)

\begin{tabular}{|c|c|}
\hline \multicolumn{2}{|l|}{ Consumers' characteristic } \\
\hline Demographic & Sex, age, family, occupation \\
\hline Geographic & $\begin{array}{l}\text { Urban city, rural city, } \\
\text { Tokyo/Osaka }\end{array}$ \\
\hline Psychographic & Life-style, personality \\
\hline Usage condition & Daily use, business use \\
\hline \multicolumn{2}{|l|}{ Consumers' response } \\
\hline Benefit response & $\begin{array}{l}\text { Quality, endurance, after sales } \\
\text { service, economical, conveni- } \\
\text { ence, swiftness }\end{array}$ \\
\hline Usage ration response & Big user, etc. \\
\hline Type of users & $\begin{array}{l}\text { Non-user, former user, first user, } \\
\text { regular user, etc. }\end{array}$ \\
\hline Frequency & Light user, heavy user, etc. \\
\hline Royalty & Absolute, non, etc. \\
\hline
\end{tabular}

1. Difference of evaluation between user and non-user for SNS

2. Difference of evaluation by attribute

3. Difference of evaluation by residential area

4. Difference of usage by each SNS

5. Difference of Psychographic characteristics between user and non-user for SNS

As it takes so many pages, we omit " 3 " stated above. We set 6 themes as follows. These are extracted from the discussion of the authors.

Theme 1: In integrated genre SNS such as Facebook, users feel interests by sharing the information on the current condition with friends.

Theme 2: Young people use SNS more frequently and dispatch much more information than seniors.

Theme 3: Company employees mainly use integrated genre SNS such as Facebook while students often use game genre SNS such as GREE.

Theme 4: Singles want to build a new network by utilizing SNS.
Theme 5: Females use Facebook, the mixi, and YouTube more frequently than males.

Theme 6: Those who like outdoors activities spread the information much more than those of indoor activity type.

\subsection{The Results of Statistical Hypothesis Testing}

The results of statistical hypothesis testing are as follows.

Theme 1: In integrated genre SNS such as Facebook, users feel interests by sharing the information of current condition with friends.

Null hypothesis: It is not clear whether users feel interests by sharing the information of current condition with friends in Integrated genre SNS such as Facebook.

Table 4. Cross tabulation result 1

\begin{tabular}{lcccc}
\hline \multirow{1}{*}{ Q61) } & \multicolumn{4}{c}{ Q4 (\%) } \\
\cline { 2 - 5 } & Facebook & mixi & Twitter & Google+ \\
\hline Think so very much & 0.537 & 0.195 & 0.170 & 0.011 \\
Slightly think so & 0.434 & 0.139 & 0.132 & 0.021 \\
Ordinary level & 0.167 & 0.064 & 0.115 & 0.064 \\
Slightly not think so & 0.294 & 0.000 & 0.088 & 0.088 \\
Do not think so & 0.087 & 0.087 & 0.174 & 0.043 \\
Sum & 0.439 & 0.150 & 0.147 & 0.024 \\
\hline & & & & \\
\hline & YouTube & Niconico $\beta$ & Ustream & GREE \\
\hline Think so very much & 0.025 & 0.014 & 0.000 & 0.005 \\
Slightly think so & 0.128 & 0.011 & 0.000 & 0.011 \\
Ordinary level & 0.179 & 0.051 & 0.000 & 0.000 \\
Slightly not think so & 0.147 & 0.206 & 0.000 & 0.059 \\
Do not think so & 0.174 & 0.087 & 0.000 & 0.087 \\
Sum & 0.087 & 0.027 & 0.000 & 0.012 \\
\hline Slightly think so & 0.021 & 0.011 & 0.028 & 0.021 \\
\hline Ordinary level & 0.115 & 0.000 & 0.013 & 0.064 \\
\hline Do not think so & 0.043 & 0.043 & 0.043 & 0.043 \\
\hline & 0.031 & 0.005 & 0.017 & 0.019 \\
\hline Mobage & Hangame & Ameba & Taberogu \\
\hline Sum & 0.016 & 0.000 & 0.008 & 0.005 \\
\hline
\end{tabular}




\begin{tabular}{lccc}
\hline & KAKAKU.com & $\begin{array}{c}\text { Ameba } \\
\text { pico }\end{array}$ & myspace \\
\hline Think so very much & 0.003 & 0.000 & 0.000 \\
Slightly think so & 0.011 & 0.004 & 0.000 \\
Ordinary level & 0.077 & 0.013 & 0.000 \\
Slightly not think so & 0.029 & 0.000 & 0.000 \\
Do not think so & 0.043 & 0.000 & 0.000 \\
Sum & 0.015 & 0.003 & 0.000 \\
\hline & \multicolumn{3}{c}{} \\
\hline & foursquare & Orkut & PowerLink \\
\hline Think so very much & 0.000 & 0.000 & 0.000 \\
Slightly think so & 0.000 & 0.000 & 0.000 \\
Ordinary level & 0.000 & 0.000 & 0.000 \\
Slightly not think so & 0.000 & 0.000 & 0.000 \\
Do not think so & 0.000 & 0.000 & 0.000 \\
Sum & 0.000 & 0.000 & 0.000 \\
\hline & \multicolumn{3}{l}{} \\
\hline & Life Shot & Miscellaneous & Total \\
\hline Think so very much & 0.000 & 0.011 & 1.000 \\
Slightly think so & 0.000 & 0.028 & 1.000 \\
Ordinary level & 0.000 & 0.077 & 1.000 \\
Slightly not think so & 0.000 & 0.000 & 1.000 \\
Do not think so & 0.000 & 0.043 & 1.000 \\
Sum & 0.000 & 0.024 & 1.000 \\
\hline
\end{tabular}

\begin{tabular}{lccc}
\hline Real number & Integrated SNS $^{*}$ & Miscellaneous $^{\dagger}$ & Sum \\
\hline Important & 537 & 109 & 646 \\
Not important & 25 & 32 & 57 \\
\hline & & & \\
\hline Expectation & Integrated SNS & Miscellaneous $^{\dagger}$ & Sum \\
\hline Important & 516.4 & 129.6 & 646 \\
Not important & 45.6 & 11.4 & 57 \\
\hline
\end{tabular}

*includes Facebook, mixi, Twitter and Google+,

${ }^{\dagger}$ the other SNS.

\begin{tabular}{lc}
\hline Statistic $\left(\chi^{2}\right.$ value $)$ & 50.627 \\
Rejection region $(1 \%$ significance level $)$ & $\mathrm{Z}>6.635$ \\
\hline${ }^{*}$ Rejection region is over 6.635 for $1 \%$ significance level and \\
3.841 for $5 \%$ significance level by 1 degree of freedom.
\end{tabular}

The null hypothesis is rejected with $1 \%$ significance level. It can be said that in Integrated genre SNS such as Facebook, users feel interests by sharing the information of current condition with friends. It can generally be said that Integrated SNS is the most suitable tool for communication compared with other genre SNS.

Theme 2: Young people use SNS more frequently and dispatch much more information than seniors.

Null hypothesis: Young people use SNS as frequently as seniors and also dispatch information similarly.
Table 5. Cross tabulation result 2-1

\begin{tabular}{|c|c|c|c|c|c|c|c|}
\hline \multirow{2}{*}{ Q2 } & \multicolumn{7}{|c|}{ Q14 (\%) } \\
\hline & $<20$ & $21-30$ & $31-40$ & $41-50$ & $51-60$ & $>60$ & Total \\
\hline $\begin{array}{l}\text { More than } 5 \\
\text { times a day }\end{array}$ & 0.383 & 0.338 & 0.197 & 0.076 & 0.007 & 0.000 & 1.000 \\
\hline $\begin{array}{l}\text { Around 3 4 } \\
\text { times a day }\end{array}$ & 0.197 & 0.421 & 0.243 & 0.112 & 0.026 & 0.000 & 1.000 \\
\hline $\begin{array}{l}\text { Around } 1 \sim 2 \\
\text { times a day }\end{array}$ & 0.112 & 0.341 & 0.324 & 0.176 & 0.047 & 0.000 & 1.000 \\
\hline $\begin{array}{l}\text { Around } 4 \sim 5 \\
\text { times a week }\end{array}$ & 0.135 & 0.173 & 0.346 & 0.269 & 0.077 & 0.000 & 1.000 \\
\hline $\begin{array}{l}\text { Around 2 3 } \\
\text { times a week }\end{array}$ & 0.157 & 0.196 & 0.373 & 0.275 & 0.000 & 0.000 & 1.000 \\
\hline $\begin{array}{l}\text { Around } 1 \\
\text { times a week }\end{array}$ & 0.061 & 0.303 & 0.303 & 0.212 & 0.121 & 0.000 & 1.000 \\
\hline $\begin{array}{l}\text { Around } 2 \sim 3 \\
\text { times a month }\end{array}$ & 0.000 & 0.444 & 0.333 & 0.111 & 0.111 & 0.000 & 1.000 \\
\hline $\begin{array}{l}\text { Around } 1 \\
\text { times a month }\end{array}$ & 0.000 & 0.167 & 0.583 & 0.167 & 0.083 & 0.000 & 1.000 \\
\hline Less than that & 0.059 & 0.294 & 0.412 & 0.118 & 0.118 & 0.000 & 1.000 \\
\hline Sum & 0.226 & 0.331 & 0.271 & 0.139 & 0.033 & 0.000 & 1.000 \\
\hline Real nu & imber & & $<40$ & & $>40$ & & um \\
\hline More than ol & nce a d & & 529 & & 83 & & 612 \\
\hline Else & & & 122 & & 52 & & 174 \\
\hline Expecta & ation & & $<40$ & & $>40$ & & um \\
\hline More than or & nce a d & & 506.9 & & 105.1 & & 612 \\
\hline Else & & & 144.1 & & 29.9 & & 174 \\
\hline Statistic $\left(\chi^{2}\right.$ ve & alue) & & & & & & \\
\hline Rejection reg & gion(1 & o signif & ficance & level) & & $Z>6$. & 635 \\
\hline
\end{tabular}

Table 6. Cross tabulation result 2-2

\begin{tabular}{|c|c|c|c|c|c|c|c|}
\hline \multirow{2}{*}{ Q8 } & \multicolumn{7}{|c|}{ Q14 (\%) } \\
\hline & $<20$ & $21 \sim 30$ & $31 \sim 40$ & $41 \sim 50$ & $51 \sim 60$ & $>60$ & Total \\
\hline Every time & 0.304 & 0.304 & 0.278 & 0.101 & 0.013 & 0.000 & 1.000 \\
\hline Frequently & 0.251 & 0.371 & 0.246 & 0.114 & 0.017 & 0.000 & 1.000 \\
\hline Sometimes & 0.213 & 0.362 & 0.253 & 0.129 & 0.043 & 0.000 & 1.000 \\
\hline Scarcely & 0.152 & 0.273 & 0.303 & 0.235 & 0.038 & 0.000 & 1.000 \\
\hline Never & 0.231 & 0.212 & 0.385 & 0.115 & 0.058 & 0.000 & 1.000 \\
\hline Sum & 0.221 & 0.333 & 0.271 & 0.140 & 0.034 & 0.000 & 1.000 \\
\hline \multicolumn{3}{|c|}{ Real number } & $<40$ & \multicolumn{2}{|c|}{$>40$} & \multicolumn{2}{|c|}{ Sum } \\
\hline \multicolumn{2}{|c|}{ Frequently } & & 222 & \multicolumn{2}{|r|}{32} & \multicolumn{2}{|c|}{254} \\
\hline \multicolumn{2}{|l|}{ Else } & & 139 & \multicolumn{2}{|r|}{45} & \multicolumn{2}{|c|}{184} \\
\hline \multicolumn{3}{|c|}{ Expectation } & $<40$ & \multicolumn{2}{|c|}{$>40$} & \multicolumn{2}{|c|}{ Sum } \\
\hline \multicolumn{2}{|c|}{ Frequently } & & 209.3 & \multicolumn{2}{|c|}{44.7} & \multicolumn{2}{|c|}{254} \\
\hline \multicolumn{2}{|l|}{ Else } & & 151.7 & \multicolumn{2}{|c|}{32.3} & \multicolumn{2}{|c|}{184} \\
\hline \multicolumn{6}{|c|}{$\operatorname{Statistic}\left(\chi^{2}\right.$ value $)$} & \multicolumn{2}{|c|}{10.436} \\
\hline \multicolumn{6}{|c|}{ Rejection region( $1 \%$ significance level) } & \multicolumn{2}{|c|}{$Z>6.635$} \\
\hline
\end{tabular}


The null hypothesis is rejected with $1 \%$ significance level. It can be said that young people use SNS more frequently and dispatch much more information than seniors. The SNS market is growing owing to the prevailing smartphones for one reason. com Score in the United States reported that the smartphone users in Japan consist of the following division of generations: 18$24,19.4 \%$; 25 34, 25.6\%; 35 44, 22.7\%; 45 54, 12.5\%; $55 \sim 64,8.6 \%$; in which $45 \%$ are $18 \sim 35$ generation.

Theme 3: Company employees mainly use Integrated genre SNS such as Facebook while students often use game genre SNS such as GREE.

Null hypothesis: It cannot necessarily be said that company employees mainly use Integrated genre SNS such as Facebook while students often use game genre SNS such as GREE.

Table 7. Cross tabulation result 3

\begin{tabular}{lcccc}
\hline \multirow{2}{*}{\multicolumn{1}{c}{ Q15 }} & \multicolumn{4}{c}{ Q4 (\%) } \\
\cline { 2 - 5 } \multicolumn{1}{c}{} & Facebook & GREE & mixi & Twitter \\
\hline Student & 0.188 & 0.020 & 0.270 & 0.223 \\
Company employee & 0.800 & 0.000 & 0.100 & 0.100 \\
Government employee & 0.530 & 0.010 & 0.080 & 0.134 \\
School teacher/staff & 0.667 & 0.000 & 0.111 & 0.000 \\
Clerk of organization & 0.500 & 0.000 & 0.286 & 0.071 \\
Independents & 0.725 & 0.000 & 0.075 & 0.025 \\
Temporary employee & 0.636 & 0.000 & 0.091 & 0.000 \\
Part-timers & 0.500 & 0.000 & 0.088 & 0.059 \\
Miscellaneous & 0.615 & 0.000 & 0.154 & 0.077 \\
Sum & 0.438 & 0.011 & 0.148 & 0.145 \\
\hline
\end{tabular}

\begin{tabular}{lccc}
\hline & Google + & YouTube & Niconico $\beta$ \\
\hline Student & 0.031 & 0.105 & 0.063 \\
Company employee & 0.000 & 0.000 & 0.000 \\
Government employee & 0.023 & 0.088 & 0.013 \\
School teacher/staff & 0.000 & 0.074 & 0.000 \\
Clerk of organization & 0.000 & 0.071 & 0.000 \\
Independents & 0.000 & 0.125 & 0.000 \\
Temporary employee & 0.000 & 0.091 & 0.000 \\
Part-timers & 0.118 & 0.088 & 0.000 \\
Miscellaneous & 0.000 & 0.000 & 0.000 \\
Sum & 0.027 & 0.092 & 0.027 \\
\hline
\end{tabular}

\begin{tabular}{lcccc}
\hline & Ustream & Mobage & Hangame & Ameba \\
\hline Student & 0.000 & 0.035 & 0.012 & 0.012 \\
Company employee & 0.000 & 0.000 & 0.000 & 0.000 \\
Government employee & 0.000 & 0.034 & 0.003 & 0.008 \\
School teacher/staff & 0.000 & 0.000 & 0.000 & 0.000 \\
Clerk of organization & 0.000 & 0.071 & 0.000 & 0.000 \\
Independents & 0.000 & 0.000 & 0.000 & 0.025 \\
Temporary employee & 0.000 & 0.091 & 0.000 & 0.000 \\
Part-timers & 0.000 & 0.000 & 0.000 & 0.118 \\
Miscellaneous & 0.000 & 0.000 & 0.000 & 0.154 \\
Sum & 0.000 & 0.030 & 0.005 & 0.016 \\
\hline
\end{tabular}

\begin{tabular}{|c|c|c|c|c|}
\hline & Taberogu & \multicolumn{2}{|c|}{$\begin{array}{l}\text { KAKAKU. } \\
\text { com }\end{array}$} & $\begin{array}{l}\text { Ameba } \\
\text { pico }\end{array}$ \\
\hline Student & 0.004 & \multicolumn{2}{|c|}{0.000} & 0.008 \\
\hline Company employee & 0.000 & \multicolumn{2}{|c|}{0.000} & 0.000 \\
\hline Government employee & 0.028 & \multicolumn{2}{|c|}{0.021} & 0.000 \\
\hline School teacher/staff & 0.074 & \multicolumn{2}{|c|}{0.074} & 0.000 \\
\hline Clerk of organization & 0.000 & \multicolumn{2}{|c|}{0.000} & 0.000 \\
\hline Independents & 0.000 & \multicolumn{2}{|c|}{0.025} & 0.000 \\
\hline Temporary employee & 0.091 & \multicolumn{2}{|c|}{0.000} & 0.000 \\
\hline Part-timers & 0.000 & \multicolumn{2}{|c|}{0.029} & 0.000 \\
\hline Miscellaneous & 0.000 & \multicolumn{2}{|c|}{0.000} & 0.000 \\
\hline \multirow[t]{2}{*}{ Sum } & 0.019 & \multicolumn{2}{|c|}{0.015} & 0.003 \\
\hline & $\begin{array}{c}\text { My } \\
\text { space }\end{array}$ & $\begin{array}{c}\text { Four } \\
\text { square }\end{array}$ & Orkut & $\begin{array}{c}\text { Power } \\
\text { Link }\end{array}$ \\
\hline Student & 0.000 & 0.000 & 0.000 & 0.000 \\
\hline Company employee & 0.000 & 0.000 & 0.000 & 0.000 \\
\hline Government employee & 0.000 & 0.000 & 0.000 & 0.000 \\
\hline School teacher/staff & 0.000 & 0.000 & 0.000 & 0.000 \\
\hline Clerk of organization & 0.000 & 0.000 & 0.000 & 0.000 \\
\hline Independents & 0.000 & 0.000 & 0.000 & 0.000 \\
\hline Temporary employee & 0.000 & 0.000 & 0.000 & 0.000 \\
\hline Part-timers & 0.000 & 0.000 & 0.000 & 0.000 \\
\hline Miscellaneous & 0.000 & 0.000 & 0.000 & 0.000 \\
\hline \multirow[t]{2}{*}{ Sum } & 0.000 & 0.000 & 0.000 & 0.000 \\
\hline & Life shot & \multicolumn{2}{|c|}{ Miscellaneous } & Total \\
\hline Student & 0.000 & \multicolumn{2}{|c|}{0.031} & 1.000 \\
\hline Company employee & 0.000 & \multicolumn{2}{|c|}{0.000} & 1.000 \\
\hline Government employee & 0.000 & \multicolumn{2}{|c|}{0.028} & 1.000 \\
\hline School teacher/Staff & 0.000 & \multicolumn{2}{|c|}{0.000} & 1.000 \\
\hline Clerk of organization & 0.000 & \multicolumn{2}{|c|}{0.000} & 1.000 \\
\hline Independents & 0.000 & \multicolumn{2}{|c|}{0.000} & 1.000 \\
\hline Temporary employee & 0.000 & \multicolumn{2}{|c|}{0.000} & 1.000 \\
\hline Part-timers & 0.000 & \multicolumn{2}{|c|}{0.000} & 1.000 \\
\hline Miscellaneous & 0.000 & \multicolumn{2}{|c|}{0.000} & 1.000 \\
\hline Sum & 0.000 & \multicolumn{2}{|c|}{0.024} & 1.000 \\
\hline Real number & ntegratedSNS & \multicolumn{2}{|c|}{ Miscellaneous } & Sum \\
\hline Company employee & 297 & \multicolumn{2}{|c|}{90} & 387 \\
\hline Miscellaneous & 303 & & 102 & 405 \\
\hline Expectation & itegrated SNS & $\mathrm{S}^{*}$ Misce & llaneous $^{\dagger}$ & Sum \\
\hline Company Employee & 293.2 & & 3.8 & 387 \\
\hline Miscellaneous & 306.8 & & 8.2 & 405 \\
\hline $\begin{array}{l}\text { *includes Facebook, m } \\
\text { SNS stated above. }\end{array}$ & lixi, Twitter a & and $\mathrm{C}$ & ogle+, & nd ${ }^{\dagger}$ other \\
\hline Statistic $\left(\chi^{2}\right.$ value $)$ & & & 0.39 & \\
\hline Rejection region $(5 \% \mathrm{si}$ & ignificance les & evel) & $Z>3$. & 841 \\
\hline
\end{tabular}




\begin{tabular}{|c|c|c|c|}
\hline Real number & Game genre SNS & Miscellaneous $^{\dagger}$ & Sum \\
\hline Student & 17 & 239 & 256 \\
\hline Miscellaneous & 20 & 516 & 536 \\
\hline Expectation & Game genre SNS ${ }^{*}$ & Miscellaneous $\dagger$ & Sum \\
\hline Student & 12.0 & 244.0 & 256 \\
\hline Miscellaneous & 25.0 & 511.0 & 536 \\
\hline \multicolumn{4}{|c|}{$\begin{array}{l}* \text { includes GREE, Mobage, and Hangame, }{ }^{\dagger} \text { other SNS stated } \\
\text { above. }\end{array}$} \\
\hline \multicolumn{2}{|c|}{ Statistic $\left(\chi^{2}\right.$ value $)$} & \multicolumn{2}{|c|}{3.235} \\
\hline \multicolumn{3}{|c|}{ Rejection region $(5 \%$ significance level) } & $Z>3.841$ \\
\hline
\end{tabular}

The null hypothesis is not rejected. It cannot necessarily be said that company employees mainly use Integrated genre SNS such as Facebook while students of the use game genre SNS such as GREE. We can observe that Facebook, which is one of the Integrated genre SNS, is used by the people of various type occupations.

Theme 4: Singles want to build a new network by utilizing SNS.

Null hypothesis: Singles do not necessarily want to build a new network by utilizing SNS.

Table 8. Cross tabulation result 4

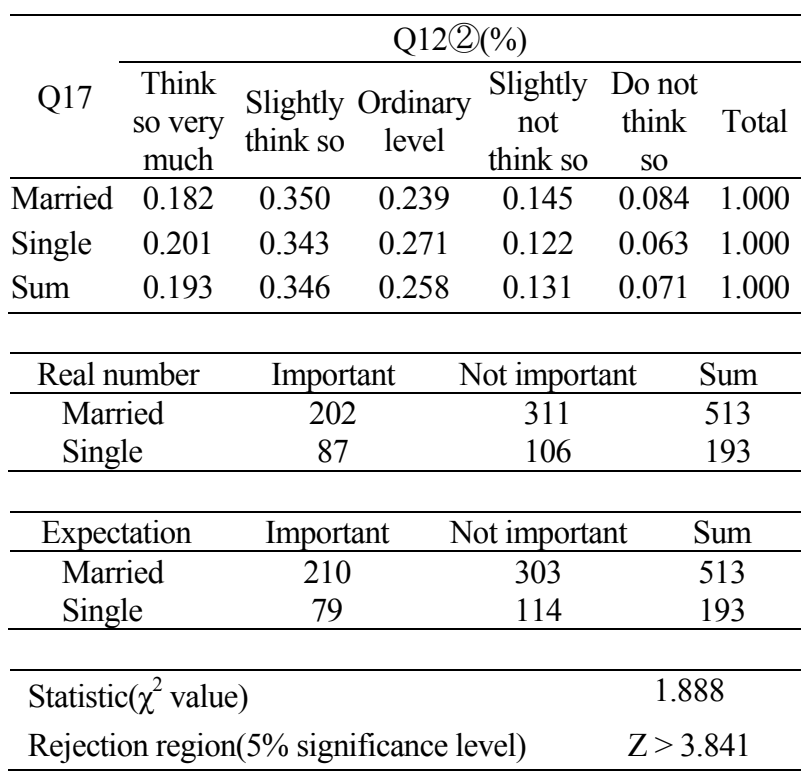

The null hypothesis is not rejected. It can be said that singles do not necessarily want to build a new network by utilizing SNS.

By another analysis, we could get the result that those who esteem getting acquainted with the friends of the opposite sex by SNS were rather few. It has few relationships with those who esteem building a new network by SNS whether they are single or married.
Theme 5: Females use Facebook more frequently than males, and the same is true with the mixi and YouTube.

Null hypothesis: Females do not use Facebook more frequently than males, nor do they use the mixi and You Tube more frequently than males.

Table 9. Cross tabulation result 5

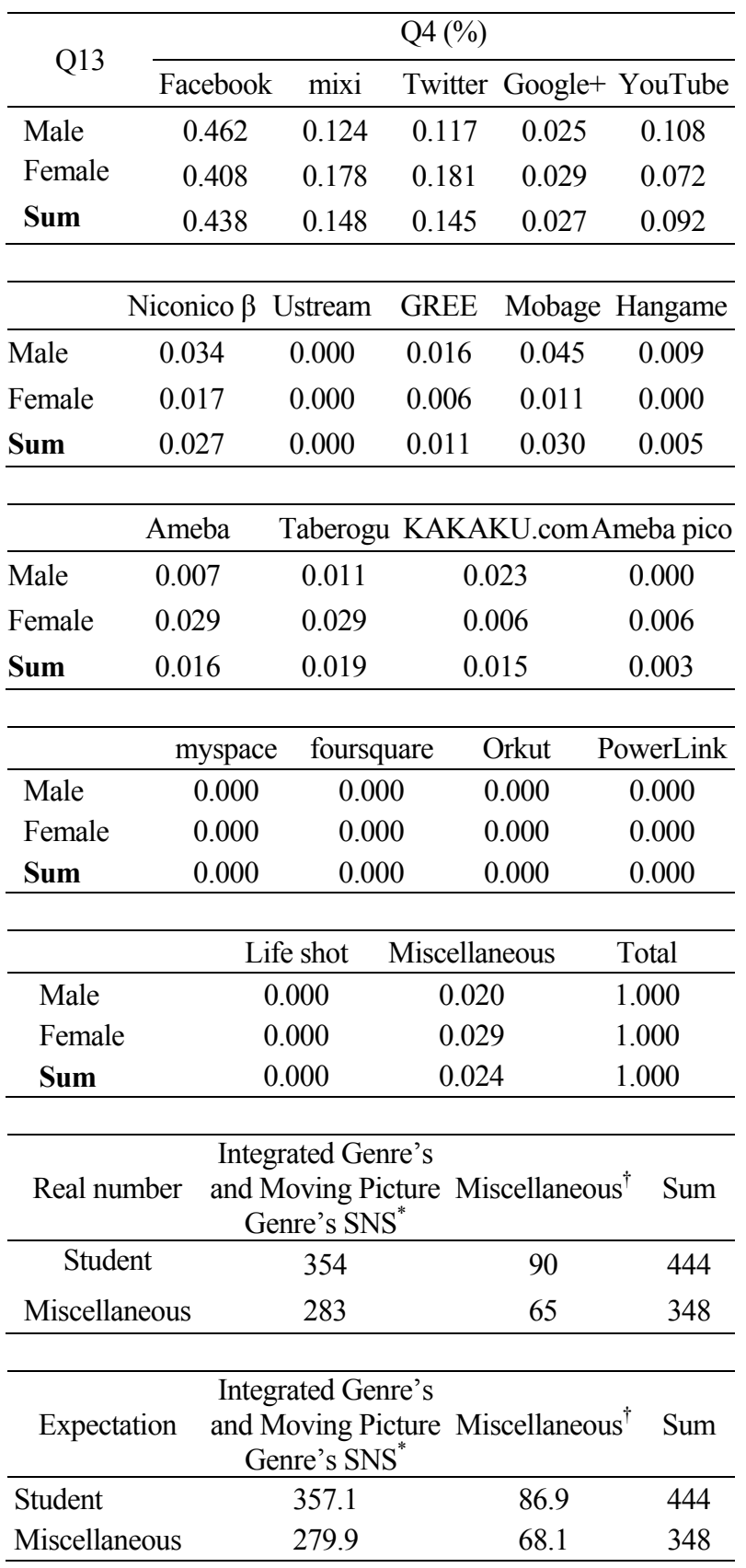

* includes Facebook, mixi, Twitter, Google+, YouTube, Niconico $\beta$, and Ustream, $\dagger$ the other SNS.

\begin{tabular}{lc}
\hline Statistic $\left(\chi^{2}\right.$ value $)$ & 0.313 \\
Rejection region(5\% significance level $)$ & $\mathrm{Z}>3.841$ \\
\hline
\end{tabular}


The null hypothesis is not rejected. Females do not use Facebook more frequently than males, nor do they use the mixi and YouTube more frequently than males.

Theme 6: Those who like outdoors activities spread the information much more than those of indoor activity type.

Null hypothesis: It cannot necessarily be said that those who like outdoors activities spread the information much more than those of indoor activity type.

Table 10. Cross tabulation result 6

\begin{tabular}{|c|c|c|c|}
\hline \multirow{2}{*}{ Q21 } & \multicolumn{3}{|c|}{ Q8 (\%) } \\
\hline & Every time & Frequently & Sometimes \\
\hline Outdoor & 0.093 & 0.195 & 0.472 \\
\hline Indoor & 0.101 & 0.237 & 0.430 \\
\hline Cannot choose either & 0.106 & 0.235 & 0.426 \\
\hline \multirow[t]{2}{*}{ Sum } & 0.101 & 0.223 & 0.441 \\
\hline & Scarcely & Never & Total \\
\hline Outdoor & 0.183 & 0.057 & 1.000 \\
\hline Indoor & 0.158 & 0.075 & 1.000 \\
\hline Cannot choose either & 0.165 & 0.068 & 1.000 \\
\hline Sum & 0.168 & 0.066 & 1.000 \\
\hline Real number & Frequently & Else & Sum \\
\hline Outdoor & 71 & 77 & 148 \\
\hline Indoor & 59 & 53 & 112 \\
\hline Expectation & Frequently & Else & Sum \\
\hline Outdoor & 74 & 74 & 148 \\
\hline Indoor & 56 & 56 & 112 \\
\hline $\operatorname{Statistic}\left(\chi^{2}\right.$ value $)$ & & & 0.565 \\
\hline Rejection region $(5 \%$ & o significance 1 & evel) & $Z>3.841$ \\
\hline
\end{tabular}

The null hypothesis is not rejected. It cannot necessarily be said that those who like outdoors activities spread the information much more than those of indoor activity type. Indoor activity type persons would use SNS on the PCs.

\section{BAYESIAN NETWORK ANALYSIS}

\subsection{Confirmation of Hypothesis by Utilizing Bayesi an Network}

Now, we examine the probabilistic inference of Bayesian network by picking up Hypothesis 1 stated above. Set "Facebook" as a parent node and "Relationship" as a child node.

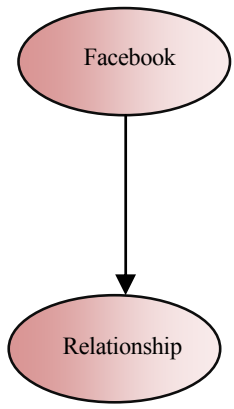

Figure 1. Node and parameter (Hypothesis 1).

As a result, we can see rather high value in Conditional Probability Table (CPT) (posterior probability in Table 11.) for Facebook "Use" and Relationship "Important". This means that users of Facebook are the majority in these questionnaire answerers and they think it important for having relationships.

Building the model with Demographic, Geographic and Psychographic items, we show that SNS can be utilized for further effective marketing in the next section.

Table 11. Built model

\begin{tabular}{llcc}
\hline \multirow{2}{*}{ Node } & Parameter & $\begin{array}{c}\text { Prior } \\
\text { probability }\end{array}$ & $\begin{array}{c}\text { Posterior } \\
\text { probability }\end{array}$ \\
\cline { 3 - 4 } Facebook & Use & 0.6335 & Facebook \\
\hline \multirow{5}{*}{ Relationship } & Not use & 0.3665 & $\mathbf{1 . 0 0 0 0}$ \\
& Important & 0.0000 \\
& Ordinary level & 0.1019 & 0.0679 \\
& Not important & 0.0739 & 0.0379 \\
\hline
\end{tabular}

\subsection{Model Structure}

In constructing Bayesian network, it is required to set an outline of the model reflecting the causal relationship among groups of items. Concept chart in this case is exhibited in Figure 2.

Haga and Motomura (2005) restricted the range of search to the following 5 stages while building the model.

(1) Selection of variables

(2) Grouping the variables

(3) Setting the search range for variable groups

(4) Setting the search range within the variable group

(5)Building the total structure

She found that it makes possible to interpret the model easily and to forecast the future activities of variables effectively.

We refer to this sample and build a model where cause and effect relationship is assumed by the order of (I) Purchaser $\Rightarrow$ (II) Extroversion and Usage condition $\Rightarrow$ (III) Purpose for Usage $\Rightarrow$ (IV)SNS. This means that (III) Purpose for Usage for (IV)SNS is influenced by (II) Extroversion and Usage condition, and one's sense of value for these is influenced by the (I) Purchaser. 


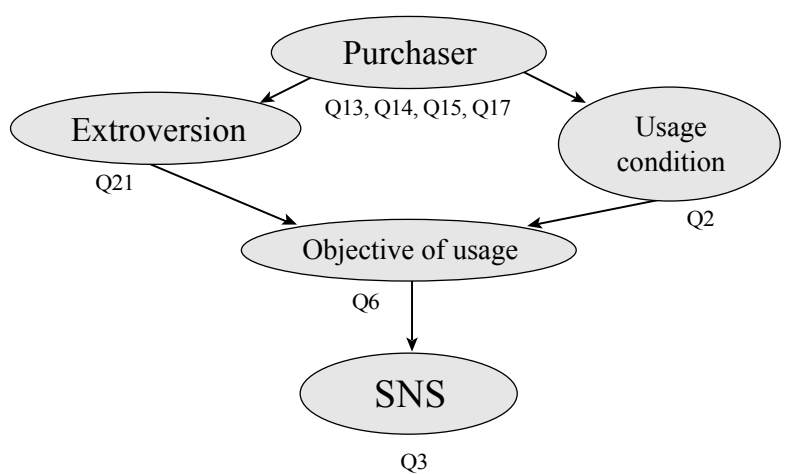

Figure 2. Node and parameter (source: Takahashi et al, 2008; revised by the writer).

We used BAYONET software (http://www.msi.co. jp/BAYONET/). When plural nodes exist in the same group, the causal relationship is hard to set a priori. In that case, the BAYONET system sets the sequence automatically utilizing AIC standard. Node and parameter of Figure 3 are exhibited in Table 12.

To decrease the number of nodes, "Think so very much" and "Slightly think so" are condensed into one. So are "Do not think so" and "Slightly not think so." We have chosen 4 sites from Integrated Genre's SNS and 1 site each from Blog Genre SNS, Moving Picture Genre SNS, genre SNS and game genre SNS.

\section{SENSITIVITY ANALYSIS}

Bayesian network calculates CPT after inputting the data. Sensitivity analysis of Bayesian network is executed mainly by the following two methods.

\section{A. Change the value in CPT}

Set the certain value in CPT and calculate again. Then

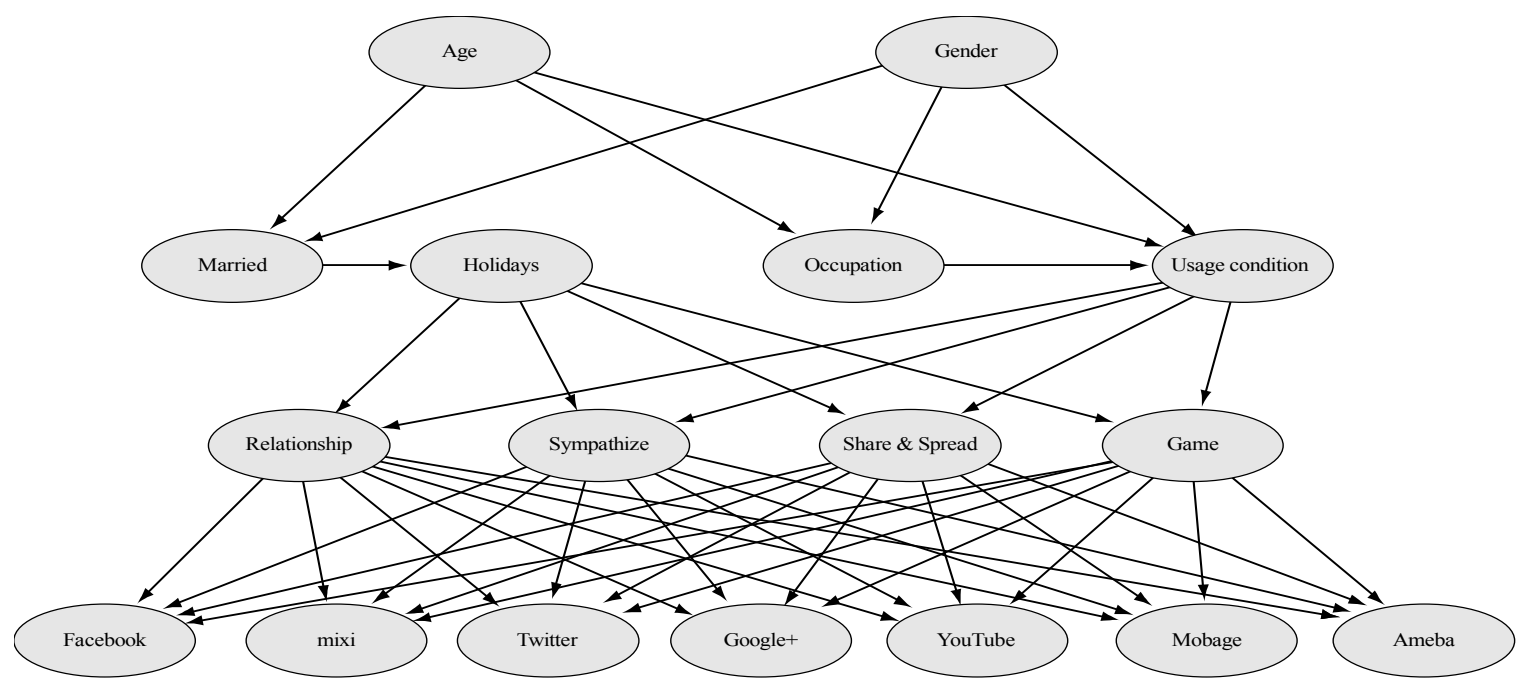

Figure 3. Builtmodel.

Table 12. Node and parameter

\begin{tabular}{|c|c|c|c|c|c|c|c|}
\hline \multirow{2}{*}{ Group name } & \multirow{2}{*}{ Node in group } & \multicolumn{6}{|c|}{ Parameter } \\
\hline & & 1 & 2 & 3 & 4 & 5 & 6 \\
\hline \multirow[t]{4}{*}{ Purchaser } & Gender & Male & Female & - & - & - & - \\
\hline & Age & $<30$ & $<50$ & $>50$ & - & - & - \\
\hline & Occupation & Student & $\begin{array}{l}\text { Company } \\
\text { employee }\end{array}$ & $\begin{array}{l}\text { School teach- } \\
\text { er/staff }\end{array}$ & Independents & Part-timers & Others \\
\hline & Married & Married & Single & - & - & - & - \\
\hline Usage condition & Usage condition & $\begin{array}{l}\text { More than } 5 \\
\text { times a day }\end{array}$ & $\begin{array}{l}\text { More than } 1 \\
\text { times a day }\end{array}$ & $\begin{array}{l}\text { More than } 1 \\
\text { times a week }\end{array}$ & Less than that & - & - \\
\hline $\begin{array}{l}\text { Purpose for } \\
\text { Usage }\end{array}$ & $\begin{array}{l}\text { Relationship, sympathize, } \\
\text { share and spread, game }\end{array}$ & Important & $\begin{array}{l}\text { Ordinary } \\
\text { level }\end{array}$ & $\begin{array}{c}\text { Not } \\
\text { important }\end{array}$ & - & - & - \\
\hline Extroversion & Holidays & Outdoor & Indoor & $\begin{array}{c}\text { Cannot } \\
\text { choose either }\end{array}$ & - & - & - \\
\hline SNS & $\begin{array}{l}\text { Facebook, mixi, Twitter, } \\
\text { Google+, YouTube, } \\
\text { Mobage, Ameba }\end{array}$ & Use & Do not use & - & - & - & - \\
\hline
\end{tabular}


the back propagation is executed and posterior probability is calculated. From the difference of prior probability and posterior probability, its influence can be observed.

\section{B. Add new input}

If the data is repeatedly inputted, then it becomes a kind of reinforcement learning, in which repeated data are stressed. Posterior probability is calculated from the new coming data (repeated data). Then the influence can be observed by comparing prior probability with posterior probability.

We use A. method and set evidence 1.0 to "Use" for each node in group (Facebook, mixi, Twitter, Google+, YouTube, Mobage, Ameba), then calculate by the back propagation method. After that, we obtain posterior probability shown in Table 13. If it goes up higher than prior probability, then we can seethe positive influence by "Use" as we have set evidence to the utmost 1.0. If it goes down, then we can see that it has a negative influ- ence in "Use", i.e., "do not use" would increase.

The result of Hypothesis testing for Hypothesis 1 is as follows. "It can be said that in Integrated Genre SNS such as Facebook, users feel interests by sharing the information of current condition with friends." In the Bayesian network analysis, Evidence 1.0 is set to "Use" in the item of "Facebook" and to "Important" in those of "Relationship." Prior probability and posterior probability is shown in Tables13 and 14.

Hatched parts are the items where posterior probability has increased. From the result, we can observe the following feature and/or characteristics.

"Outdoor typed Single male of Student/School teacher/Independents/Part-timers who are under 30 andlike Facebook use mixi/Twitter/Google+/Mobage/Ameba more than 1 time a week and esteem "Share and spread".

But hatched parts include subtle changes. Therefore, we calculate the odds ratio. It is often seen that the change of the probability becomes small when the hierarchical data cluster is distant. Posterior probability is calculated in the back propagation method. It is often

Table 13. Odds ratio for "Use"

\begin{tabular}{|c|c|c|c|c|c|c|}
\hline Node & Parameter & $\begin{array}{c}\text { Prior } \\
\text { probability } \\
\end{array}$ & $\begin{array}{l}\text { Common } \\
\text { logarithm }\end{array}$ & $\begin{array}{c}\text { Posterior } \\
\text { Probability (Use) }\end{array}$ & $\begin{array}{l}\text { Common } \\
\text { logarithm }\end{array}$ & Odds ratio \\
\hline \multirow[t]{2}{*}{ Gender } & Male & 0.5918 & 0.7642 & 0.5923 & 0.7723 & 1.0953 \\
\hline & Female & 0.4082 & 0.6107 & 0.4077 & 0.6096 & 0.9908 \\
\hline \multirow[t]{3}{*}{ Age } & $<30$ & 0.4768 & 0.6776 & 0.4797 & 0.6803 & 1.0251 \\
\hline & $<50$ & 0.4487 & 0.6513 & 0.4467 & 0.6493 & 0.9826 \\
\hline & $>50$ & 0.0745 & 0.8722 & 0.0735 & 0.8663 & 0.9014 \\
\hline \multirow[t]{5}{*}{ Occupation } & Student & 0.2672 & 0.4265 & 0.2699 & 0.4298 & 1.0273 \\
\hline & Company employee & 0.5417 & 0.7324 & 0.5175 & 0.7135 & 0.8280 \\
\hline & School teacher/staff & 0.0399 & 0.601 & 0.0421 & 0.6232 & 1.2057 \\
\hline & Independents & 0.0417 & 0.6201 & 0.0459 & 0.6618 & 1.4372 \\
\hline & Part-timers & 0.0489 & 0.6893 & 0.0580 & 0.7634 & 2.1151 \\
\hline \multirow[t]{2}{*}{ Married } & Married & 0.4236 & 0.6235 & 0.4172 & 0.6201 & 0.9715 \\
\hline & Single & 0.5764 & 0.7513 & 0.5828 & 0.7649 & 1.1599 \\
\hline \multirow[t]{3}{*}{ Usage condition } & More than 5 times a day & 0.3679 & 0.5647 & 0.3120 & 0.4942 & 0.5673 \\
\hline & More than 1 times a day & 0.4083 & 0.6107 & 0.3882 & 0.5888 & 0.8332 \\
\hline & More than 1 times a week & 0.1745 & 0.2405 & 0.1940 & 0.2878 & 1.6286 \\
\hline Relationship & Important & 0.8242 & 0.9159 & 0.8361 & 0.9222 & 1.1846 \\
\hline Sympathize & Important & 0.3852 & 0.5885 & 0.3309 & 0.5185 & 0.5670 \\
\hline Share and spread & Important & 0.6140 & 0.7882 & 0.6217 & 0.7931 & 1.0610 \\
\hline Game & Important & 0.2538 & 0.4031 & 0.1972 & 0.2945 & 0.3821 \\
\hline \multirow[t]{2}{*}{ Holidays } & Outdoor & 0.3106 & 0.4914 & 0.3148 & 0.4969 & 1.0450 \\
\hline & Indoor & 0.2687 & 0.4281 & 0.2594 & 0.4133 & 0.8856 \\
\hline Facebook & Use & 0.6335 & 0.8014 & 1.0000 & - & - \\
\hline $\operatorname{mixi}$ & Use & 0.4950 & 0.6946 & 0.5310 & 0.7251 & 1.3450 \\
\hline Twitter & Use & 0.4219 & 0.6243 & 0.4556 & 0.658 & 1.3406 \\
\hline Google+ & Use & 0.1423 & 0.1523 & 0.2056 & 0.3118 & 6.3593 \\
\hline YouTube & Use & 0.5617 & 0.749 & 0.5369 & 0.7292 & 0.8143 \\
\hline Mobage & Use & 0.1159 & 0.0607 & 0.1730 & 0.238 & 23.3601 \\
\hline Ameba & Use & 0.1486 & 0.1703 & 0.2220 & 0.3464 & 6.6672 \\
\hline
\end{tabular}


Table 14. Odds ratio for "Important"

\begin{tabular}{|c|c|c|c|c|c|c|}
\hline Node & Parameter & $\begin{array}{c}\text { Prior } \\
\text { Probability }\end{array}$ & $\begin{array}{l}\text { Common } \\
\text { logarithm }\end{array}$ & $\begin{array}{c}\text { Posterior } \\
\text { Probability } \\
\text { (Important) }\end{array}$ & $\begin{array}{l}\text { Common } \\
\text { logarithm }\end{array}$ & Odds ratio \\
\hline \multirow[t]{2}{*}{ Gender } & Male & 0.5918 & 0.7642 & 0.5932 & 0.7731 & 1.1053 \\
\hline & Female & 0.4082 & 0.6107 & 0.4068 & 0.6085 & 0.9817 \\
\hline \multirow[t]{3}{*}{ Age } & $<30$ & 0.4768 & 0.6776 & 0.4931 & 0.6928 & 1.1514 \\
\hline & $<50$ & 0.4487 & 0.6513 & 0.4364 & 0.6395 & 0.9020 \\
\hline & $>50$ & 0.0745 & 0.8722 & 0.0705 & 0.8482 & 0.6703 \\
\hline \multirow[t]{5}{*}{ Occupation } & Student & 0.2672 & 0.4265 & 0.2771 & 0.4425 & 1.1391 \\
\hline & Company employee & 0.5417 & 0.7324 & 0.516 & 0.7126 & 0.8207 \\
\hline & School teacher/staff & 0.0399 & 0.601 & 0.0409 & 0.6117 & 1.0938 \\
\hline & Independents & 0.0417 & 0.6201 & 0.0441 & 0.6444 & 1.2325 \\
\hline & Part-timers & 0.0489 & 0.6893 & 0.0557 & 0.7459 & 1.7507 \\
\hline \multirow[t]{2}{*}{ Married } & Married & 0.4236 & 0.6235 & 0.4095 & 0.6117 & 0.9049 \\
\hline & Single & 0.5764 & 0.7513 & 0.5905 & 0.7709 & 1.2407 \\
\hline \multirow[t]{3}{*}{ Usage condition } & More than 5 times a day & 0.3679 & 0.5647 & 0.3443 & 0.5366 & 0.7968 \\
\hline & More than 1 times a day & 0.4083 & 0.6107 & 0.3979 & 0.5988 & 0.9052 \\
\hline & More than 1 times a week & 0.1745 & 0.2405 & 0.1703 & 0.2304 & 0.8938 \\
\hline Relationship & Important & 0.8242 & 0.9159 & 1.0000 & - & - \\
\hline Sympathize & Important & 0.3852 & 0.5885 & 0.3729 & 0.5705 & 0.8626 \\
\hline Share and spread & Important & 0.6140 & 0.7882 & 0.5921 & 0.7723 & 0.8307 \\
\hline Game & Important & 0.2538 & 0.4031 & 0.2485 & 0.3945 & 0.9308 \\
\hline \multirow[t]{2}{*}{ Holidays } & Outdoor & 0.3106 & 0.4914 & 0.3136 & 0.4955 & 1.0333 \\
\hline & Indoor & 0.2687 & 0.4281 & 0.2651 & 0.4232 & 0.9607 \\
\hline Facebook & Use & 0.6335 & 0.8014 & 0.6593 & 0.8189 & 1.2557 \\
\hline mixi & Use & 0.4950 & 0.6946 & 0.5578 & 0.7459 & 1.6658 \\
\hline Twitter & Use & 0.4219 & 0.6243 & 0.468 & 0.6702 & 1.4956 \\
\hline Google + & Use & 0.1423 & 0.1523 & 0.1878 & 0.2718 & 4.3160 \\
\hline YouTube & Use & 0.5617 & 0.749 & 0.5421 & 0.734 & 0.8551 \\
\hline Mobage & Use & 0.1159 & 0.0607 & 0.1592 & 0.2014 & 15.2297 \\
\hline Ameba & Use & 0.1486 & 0.1703 & 0.2092 & 0.3201 & 5.2613 \\
\hline
\end{tabular}

spoken metaphorically that back propagation is a kind of wave after the collision to the wall. Therefore, it decreases greatly as it parts from the "wall." Therefore, we take common logarithm before calculating the odds ratio. The odds ratio is calculated in Tables 13 and 14.

When Evidence is set to 1.0 for "Use" in "Facebook", the Odds ratio parts for more than 2.0 are "Parttimers", "Google+", "Mobage", and "Ameba." These have strong correlation for the users of "Facebook."

When Evidence is set to 1.0 for "Important" in "Relationship", the odds ratio parts for more than 2.0 are "Google+", "Mobage", and "Ameba." These have strong correlation for those who esteem relationship. These have a similarity in having or constructing relationship with friends.

As is stated before, the change of the probability becomes small when the hierarchical data cluster is distant.

To this point, reinforcement learning, for example, may be one of the improving methods to cope with this.
As stated before, if the data is repeatedly inputted, then it becomes a kind of reinforcement learning, in which repeated data is stressed. The decrease is improved by this reinforcement learning and we can observe its influence more clearly. Another improving method is to make shallow the depth of the hierarchy of the model.

Thus, utilizing the sensitivity analysis, we can make clear the difference of usage objective and SNS site by the attributes and preference of SNS users. Sensitivity analysis can be utilized effectively for marketing by clarifying the target customer through the sensitivity analysis.

\section{CONCLUSION}

SNS has been prevailing rapidly in Japan in recent years. Facebook, mixi, and Twitter are the popular ones. These are utilized in various fields of life together with the convenient tools such as smart-phones. 
In this paper, a questionnaire investigation was executed in order to clarify the current usage condition, issues and desired functions. Difference of usage objectives and SNS sites was made clear by the attributes and preference of SNS users. These differences can be utilized effectively for marketing by clarifying the target customer through the sensitivity analysis.

Various cases should be examined hereafter.

\section{REFERENCES}

Cabinet Office, Government of Japan (2012), cited 2013 May 15, http://www.cao.go.jp.

Dentsu Inc. (2011), cited 2013 May 15, http://www.den tsu.co.jp.

Haga, M. and Motomura, Y. (2005) Marketing strategy and product development using Bayesian networks, Jinko Chino GakkaiJinko Chino KihonMondaiKenkyukai Shiryo, 60, 59-61.

ICT Research and Consulting Inc. (2012), cited 2013 May 15, Available from: http://www.ictr.co.jp.

Kotler, P. and Keller, K., Brady, M., Goodman, M., and Hansen. T. (2012), Marketing Management (2 ed.), Pearson, Harlow, UK.

Kotler, P., Kartajaya, H., and Setiawan, I. (2010), Marketing 3.0: from Products to Customers to the Human Spirit, John Wiley and Sons, Hoboken, NJ.

Neapolitan, R. E. (2004), Learning Bayesian Networks, Pearson Prentice Hall, Upper Saddle River, NJ.

Nielsen. (2012), cited 2013 May 15, http://www.nielsen. com.

Sato, N. (2011), Communication Tomorrow, ASCII Media Works Inc., Tokyo, Japan.

Suwa, H. and Ohta, T. (2010), Social media in evolution of organization and community, Journal of Japanese Society for Artificial Intelligence, 25(6), 841849.

Takahashi. A., Aoki, S., Tsuji, H., and Inoue, S. (2008), Bayesian network for future home energy consumption, Lecture Notes in Computer Science, 5243, 372-379.

Tatsuoka, K., Yoshida, T., and Munemoto, J. (2008a), Analysis on purchase behavior according to display way of merchandise by Bayesian network, Journal of Architecture and Planning (Transactions of AIJ), 73(633), 2349-2354.

Tatsuoka, K., Yoshida, T., and Munemoto, J. (2008b), Analysis on relationship between layout of displaycases ad purchase behavior by Bayesian network, Journal of Architecture and Planning (Transactions of AIJ), 73(634), 2633-2638.

Tsuji. H., Kojima, M., Takahashi, A., Nakano, M., Aoki, S., Inoue, S., Asari, K., and Mimura, E. (2008), Preference mining on future home energy consumption,
IEEE International Conference on Systems, Man and Cybernetics, Singapore, 3697-3701.

\section{APPENDIX}

\section{Questionnaire concerning the SNS}

Please answer the following questions. Please write down $\circ$ to the answering items. Plural selection is allowed for the Question 3, 5, 7, 9, 10. Select (1)-(5) of the right column for the Question 6, 11, 12 .

\section{Do you use the SNS?}

Q1 (1) YES (2) NO * If you answer "(2) NO", then proceed to Q11, please.

\section{How often do you use the SNS?}

\begin{tabular}{|l} 
Q2 (1) More than 5 times a day \\
(2) Around 3-4 times a day \\
(3) Around 1-2 times a day \\
(4) Around $4-5$ times a week \\
(5) Around 2-3 times a week \\
(6) Around 1 times a week \\
(7) Around 2-3 times a month \\
(8) Around 1 times a month 9 Less than that
\end{tabular}

\section{What kind of the SNS do you use?}

Q3 (1) Facebook (2) mixi (3) Twitter (4) Google+

(5) YouTube (6) Niconico $\beta$ (7) Ustream (8) GREE

(9) Mobage (10) Hangame (11) Ameba (12) Taberogu

(13) KAKAKU.com (14) Ameba pico (15) myspace

(16) foursquare (17) Orkut (18) PowerLink (19) Life Shot

(20) Miscellaneous ( )

\section{What kind of the SNS do you usethemost?}

\section{(}

\section{Why is it?}

Q5 (1) Able to communicate with friends and acquaintances

(2) Able to seek old friends and acquaintances

(3) Able to seek new friends and acquaintances

(4) Able to agree with/appreciate the valuable information

(5) Able to collect special and delightful information

(6) Able to browse artists/celebrities' comments

(7) Able to apply for the campaign

(8) Able to collect/put out the company's services and service information

(9) Able to collect/put out hobby and interesting information

(10) Able to post diary, tweets, moving images and pho- 
tos

(11) Able to play the game (including the online game)

(12) Miscellaneous ( )

6. What are the SNS's interesting and fascinating points?

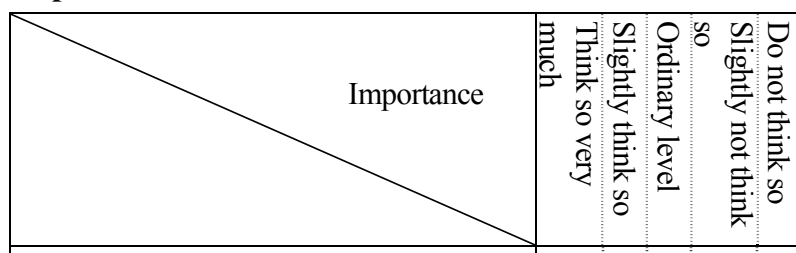

Q6 (1) Able to communicate with each other by diary and tweets

(2) Obtained much opportunities to contact with friends and acquaintances who were under rare contact

(3) Able to find new friend who has the same hobby and interest via the Net

(4) Able to get a feeling of intimacy by browsingartists/ celebrities' comments

(5) Able to collect news and information efficiently

6) Able to share sympathy by joining a group

(7) Able to share hobby and interests with friends and acquaintances

(8) Able to let others know about myself well

(9) Able to control the information for public, which is different from blog

(10) Able to retain the thinking of our own opinion and to make the record

(11) Rich online game

(12) Good for killing time

(13) Miscellaneous ( )

\section{How did you come to use the SNS?}

Q7 (1) To create a new network

(2) Agree with/ appreciate the valuable information

(3) To collect information

(4) Utilize to my business

(5) To apply for the campaign

6) To Put out and share the information

(7) Sound like fun by posting everything

(8) More easy to handle than those by phone and e-mail

(9) Suggestion by the friends and acquaintances

(10) Acquaintances and friends use them

(11) Became current topics

(12) Miscellaneous ( )
8. How often do you reply to the comments or share photos and news?

Q8 (1) Every time (2) Frequently (3) Sometimes

(4) Scarcely (5) Never

9. What kind of the SNSare you going to continue to use?

Q9 (1) Facebook (2) mixi (3) Twitter (4) Google+

(5) YouTube (6) Niconico $\beta$ (7) Ustream (8) GREE

(9) Mobage (10) Hangame (11) Ameba (12) Taberogu

(13) KAKAKU.com (14) Ameba pico (15) myspace

(16) foursquare (17) Orkut (18) PowerLink (19) Life Shot

(20) Miscellaneous ( )

\section{Why is it?}

Q10 (1) Want to enrich communication with friends and acquaintances

(2) Want to seek old friends and acquaintances

(3) Want to seek new friends and acquaintances

(4) Want to agree with/appreciate the valuable information

(5) Want to collect beneficial and delightful information

(6) Want to browse artists/celebrities' comments

(7) Want to apply for the campaign

(8) Want to collect/put out the company's services and service information

(9) Want to collect/put out hobby and interesting information

(10) Want to continue posting diary, tweets, moving images and photos

(11) Want to play the new game (including the online game)

(12) Miscellaneous ( ) 
11. Why don't you use the SNS?

Q11 (1) Do not have interest
(2) Interesting but do not know how
to use
(3) Anxious about security concern-
ing individual information
(4) Anxious about fee
(5) Surrounding people do not use
them
(6) Become poor in human com-
munication
(7) Will waste a lot of time
(8) Feel uneasy how friends and ac-
quaintances make response
(9) Cannot continue because it is
too bothering
(11) Likely to increase the spam e-
mail
(11) Miscellaneous ( )

\section{What do you expect the SNS in the future?}

Q12 (1) Make full communication with
friends and acquaintances
(2) Want to seek old friends, ac-
quaintances and new friends
(3) Encounter the friend/lover of
opposite sex
(4) Provide the valuable informa-
tion
(5) Enrich the collection of in-
formation
(6) Have a space/field for ex-
changing opinion about goods,
service and politics
(7) Gather interesting information
(8) To disclose the information of
(9imself
(9) To make perfect the security
of individual information
(10) Easiness in using
(11) Restrict the writing in board
by others
(12) Interconnection among SNS
functions
(13) Miscellaneous ( )

About yourself

\begin{tabular}{|l|}
\hline Q13 $<$ Gender $>$ (1) Male (2) Female \\
\hline Q14 $<$ Age $>$ ( ) years old \\
\hline Q15 $<$ Occupation $>$ (1) Student (2) Government Employee \\
(3) Company Employee (4) School Teacher/Staff (5) \\
Clerk of Organization (6) Independents (7) Temporary \\
Employee (8) Part-timers (9) Miscellaneous ( ) \\
\hline Q16 $<$ Address > Prefecture : ( ) City: ( ) \\
\hline Q17 $<$ Are you married?> (1) Married (2) Single \\
\hline Q18 $<$ How many children do you have?> ( ) \\
\hline Q19 $<$ Are you positive to do anything?> (1) Positive \\
(2) Somewhat positive (3) Ordinary level (4) Somewhat \\
passive (5) Not positive \\
\hline Q20 $<$ Do you like to play with many others? $>$ \\
(1) Think so very much (2) Slightly think so (3) Ordinary \\
level (4) Slightly not think so (5) Do not think so \\
\hline Q21 $<$ How do you spend holidays?> (1) Outdoor (2) Indoor \\
(3) Cannot choose either \\
\hline Q22 $<$ What is the most important thing to you?> \\
(1) Affection (2) Safety and security (3) Honor \\
(4) Clothes/Eating/House (5) Self-realization (6) Contri- \\
bution to society (7) Recognized from others (8) Miscel- \\
laneous ( ) \\
The figure below is an on-line data gathering Forth
\end{tabular}

The figure below is an on-line data gathering Form for Questionnaire Investigation.

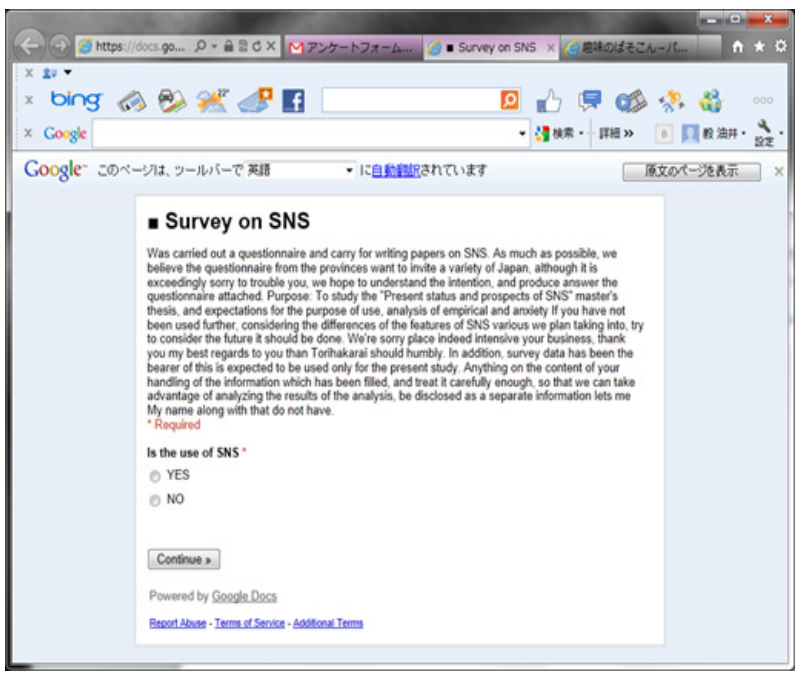

Figure A. On-line data gathering Form for Questionnaire Investigation (https://docs.google.com). 